\title{
New method for visualising discontinuities in the crystalline lens
}

\author{
R A WEALE \\ From the Department of Visual Science, Institute of Ophthalmology, University of London, Judd Street, \\ London WC1H 9 QS
}

SUMmARY When the illuminating beam of a slit-lamp is polarised and one of the viewing oculars and the camera lens are provided with crossed analysers, the image of the lens generally darkens. The effect is enhanced by the insertion of a quarter-wave plate. The contrast of lenticular discontinuities is consequently greatly accentuated. This makes it possible to quantify them.

The conventional description of a slit-lamp image is said to pick out the cornea, the 'optically' empty space of the aqueous, and the white mass of the lens. The bluish colour is attributed to light being scattered by the lens, increasingly so as age advances. Sometimes reference is made to the third and fourth Purkinje images, with the latter appearing comparatively yellow because short-wavelength radiations are preferentially scattered and/or absorbed as they traverse the lens, so leaving the complementary tint in the posterior reflexion. This picture is accentuated when a cataract is present.

However, this account harbours some unexplained observations. It is a matter of common clinical experience that the patient's vision is often subjectively far better than an objective appraisal of his lens would lead one to believe. Furthermore, Zuckerman et al.,' who studied the image-forming attributes of excised lenses with artificially produced 'cataracts', also emphasised the advanced stage of deterioration needed for the production of optically adverse consequences to be realised.

These difficulties would be resolved if it were to turn out that what appears to be light scatter may not conform to the conventional definition which entails the notion of randomness. It is well known that scattering media tend to depolarise radiation that is initially polarised, and this provides consequently a test for the optical nature of doubtful media. ${ }^{2}$ It is of course important to allow for the angle between the incident and the scattered light: the latter may contain a significant moiety of polarised light at $90^{\circ}$.

Although polarised light has been used for the study of the human eye in the past, ${ }^{3}$ the procedure Correspondence to Professor R A Weale. does not appear to have been used clinically. It has recently been found that if, in addition to being provided with a pair of crossed polaroids, a slit-lamp beam passes through a suitable quarter-wave plate which compensates for the birefringence of the cornea and is rotatable, the well-known Maltese cross $^{4}$ can be made to move across the pupil. Consequently, any part of the lens can be occluded in a manner that is quantifiable. ${ }^{5}$ One would expect occlusion to be less effective in the presence of scattering nuclei, and the fraction of real to apparent scatter indeed rises with age.

However, it is only in the eighth or ninth decade of life that the distinction between the two tends to vanish. What, then, happens if a cataract is present? Conversely, is it possible that the apparent scatter impedes the detection of optical discontinuities in the lens and that, if such scatter were reduced, we would see a more informative picture not otherwise available for diagnosis? It may be mentioned parenthetically that Peli $^{6}$ has recently used a non-crossing polarised-retarded system to enhance contrast in the corneal endothelium.

\section{Material and methods}

A Zeiss photographic slit-lamp was modified in the following manner. A piece of polaroid (axis horizontal) was placed over the illuminating beam, and one piece (axis vertical) each over the camera beam and the left ocular. In addition a rotatable quarterwave plate $(\lambda=529 \mathrm{~nm})$ was placed between the first piece of polaroid and the patient's eye. Although polaroid with a transmission factor $\mathrm{T}=22 \%$ would have been desirable, the output of the flash tube 

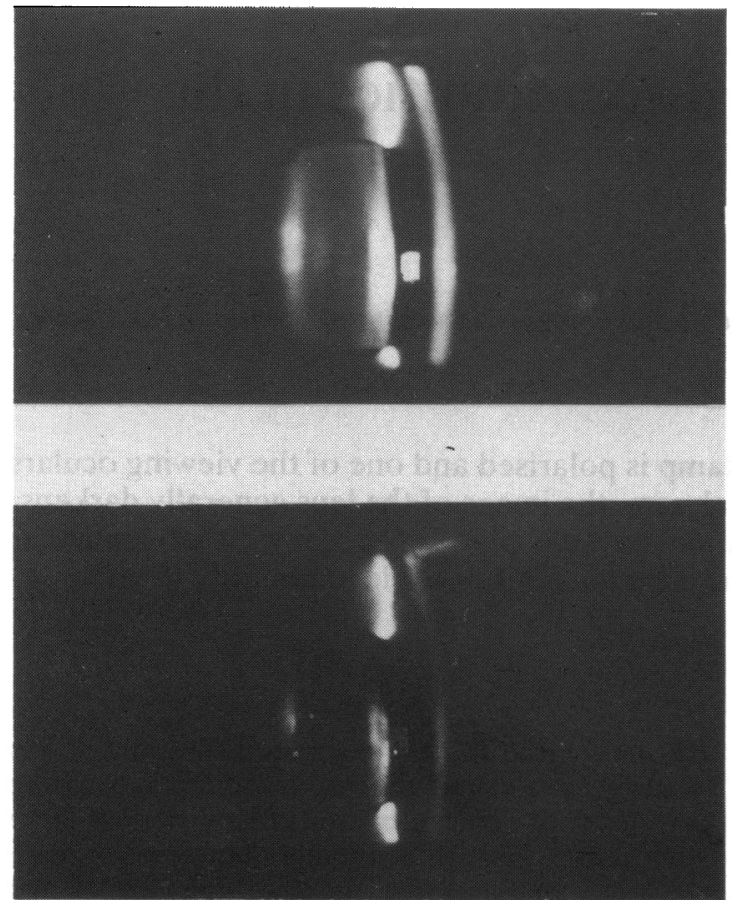

Fig. 1
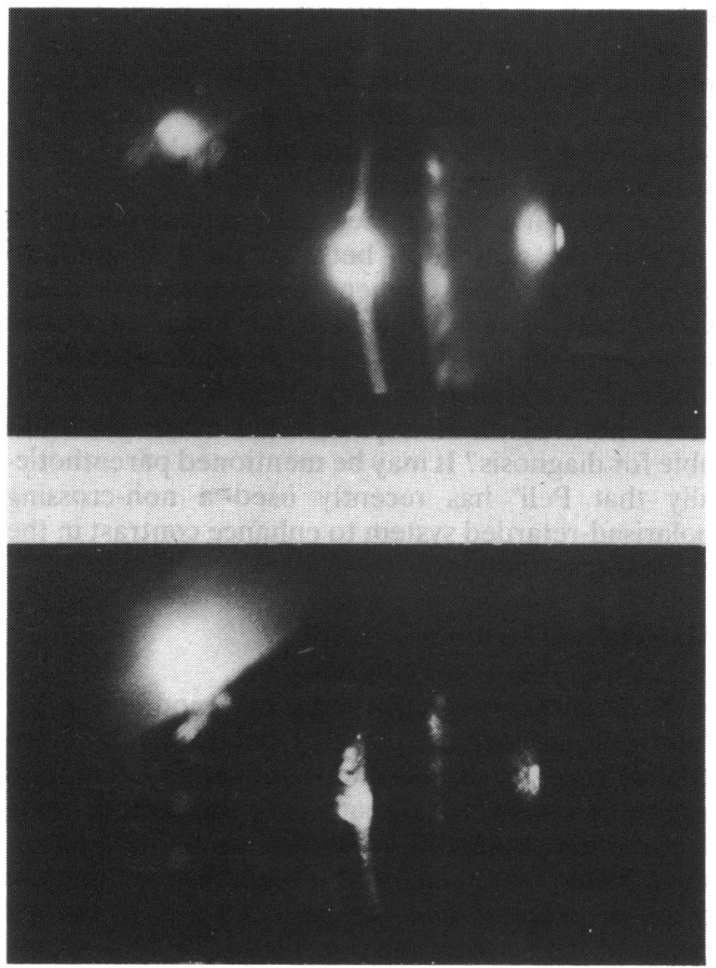

Fig. 2
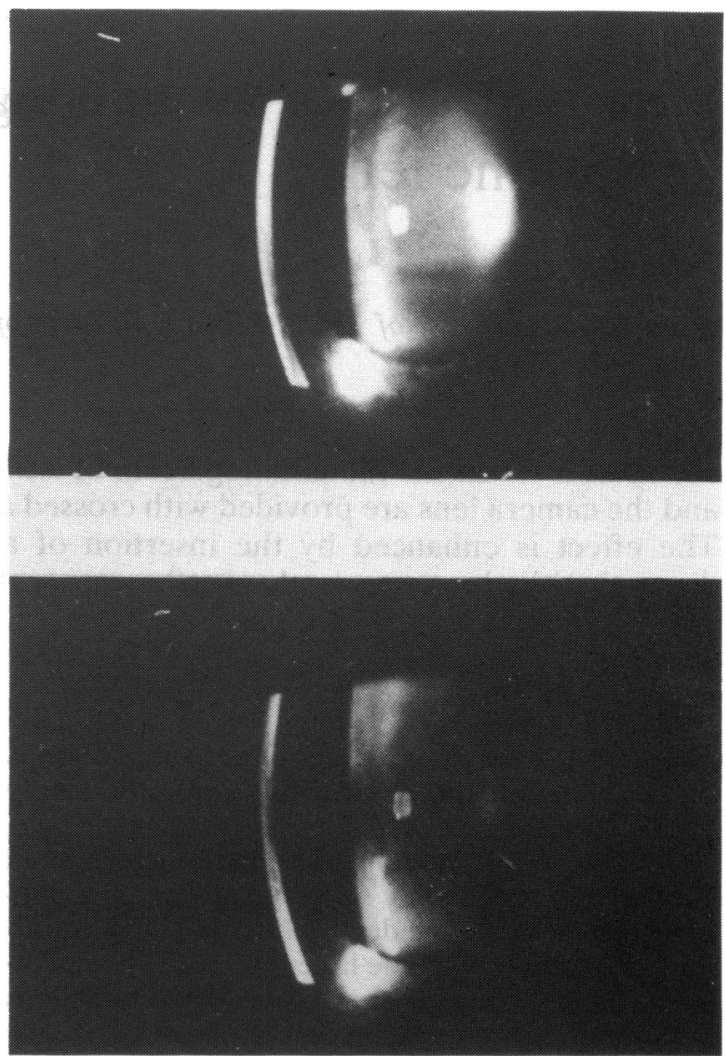

Fig. 3

made photography difficult, and therefore a transmissivity of $T=43 \%$ had to be tolerated. For clinical visual use the better type is preferable, as it enhances contrast.

For the purpose of a photographic demonstration the quarter-wave plate was rotated so as to minimise and maximise contrast in selected parts of the field. In the former case the picture approached that seen in conventional viewing, as is to be expected on theoretical grounds.'

\section{Results}

Typical comparisons as between the conventional and the more novel appearances are shown in the following illustrations. In general the conventional view is placed at the top.

Fig. 1 shows the appearance for a normal 48-yearold lens. The normal scatter in the lens and cornea is diminished in the lower picture. The lens is optically evacuated, and water-clefts, not normally visible, appear in the anterior part of the lens.

Fig. 2 shows, for another eye, not only that the 

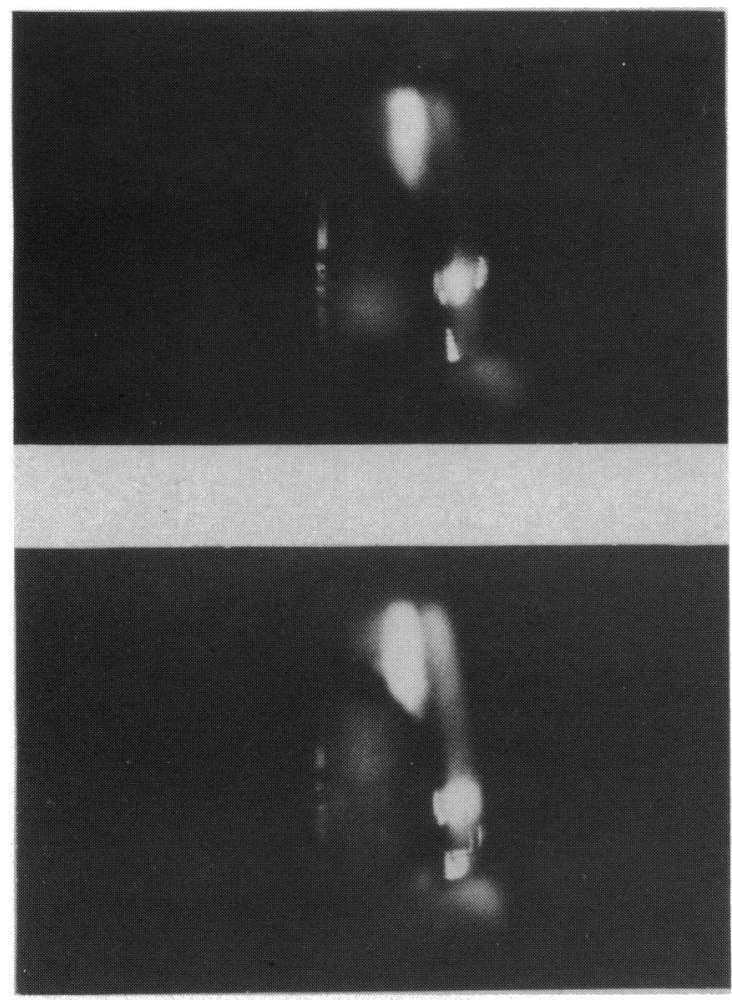

Fig. 4

clefts can be effectively isolated, but also that when the slit-lamp is focused on the cornea the corneal criss-cross drawn and explained by Koeppe ${ }^{2}$ is superposed on the image.
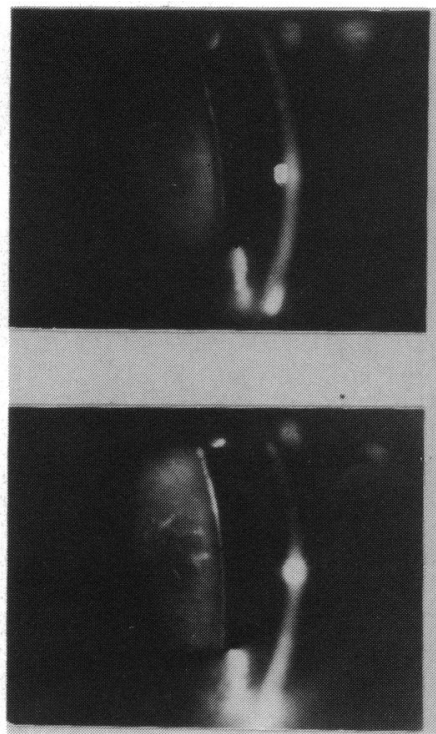

Fig. 5

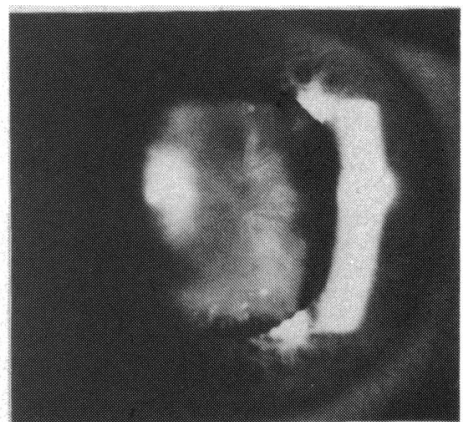

Fig. 6

Fig. 3 provides evidence in support of the view that the optical 'evacuation' of the lens fails in places of pronounced scattering agglomerates, as indicated in the lower part of the lens.

Fig. 4 illustrates not only a posterior subcapsular cataract but also shows that a nuclear cataract is
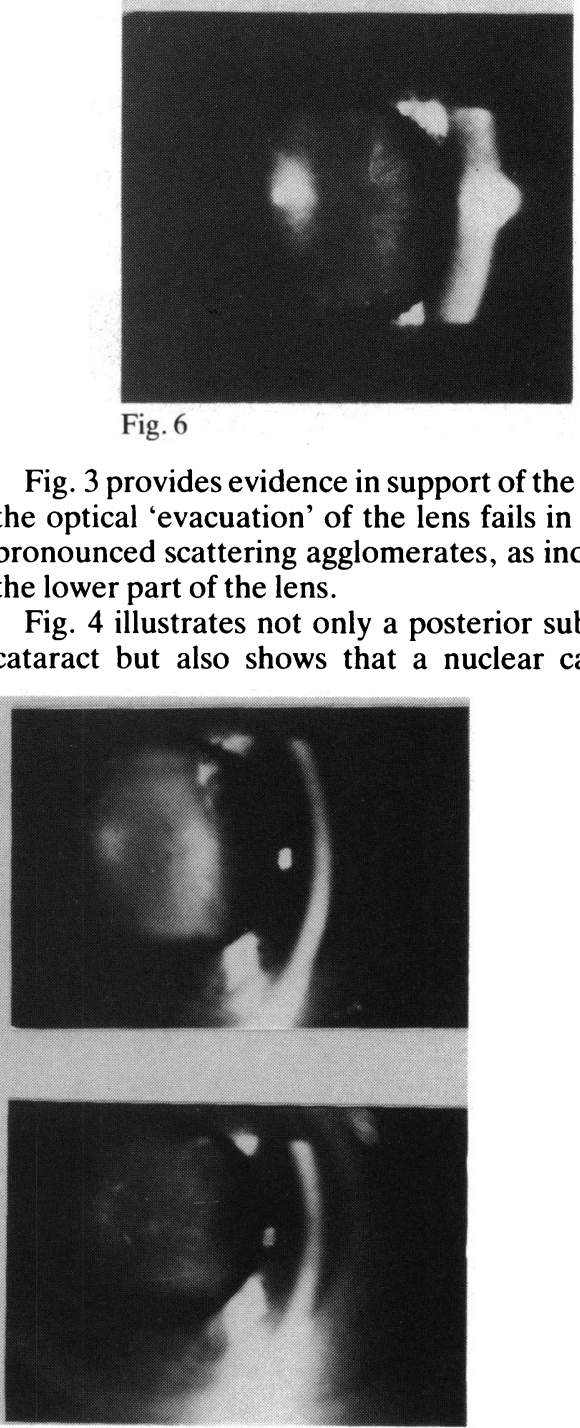

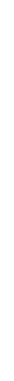




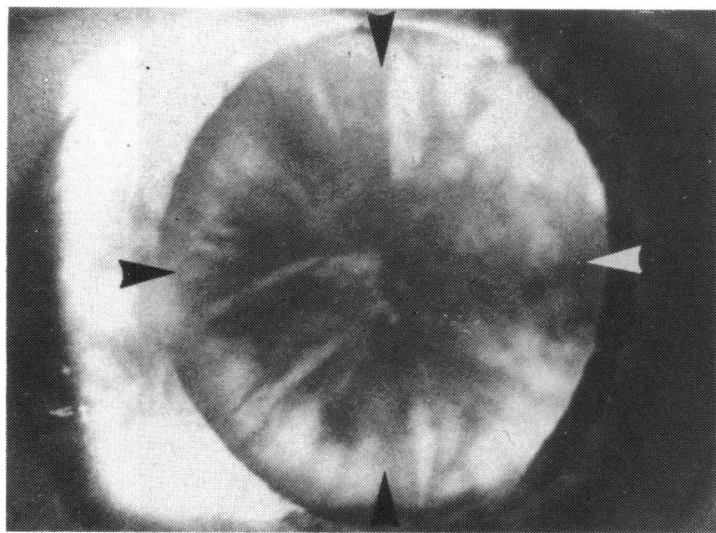

Fig. 7

present. However, rotation of the quarter-wave plate (Q) enables one to darken different parts of the lens thereby revealing water clefts (cf. lower picture). This illustration also shows that a nuclear cataract does not contain only randomly scattering discon-
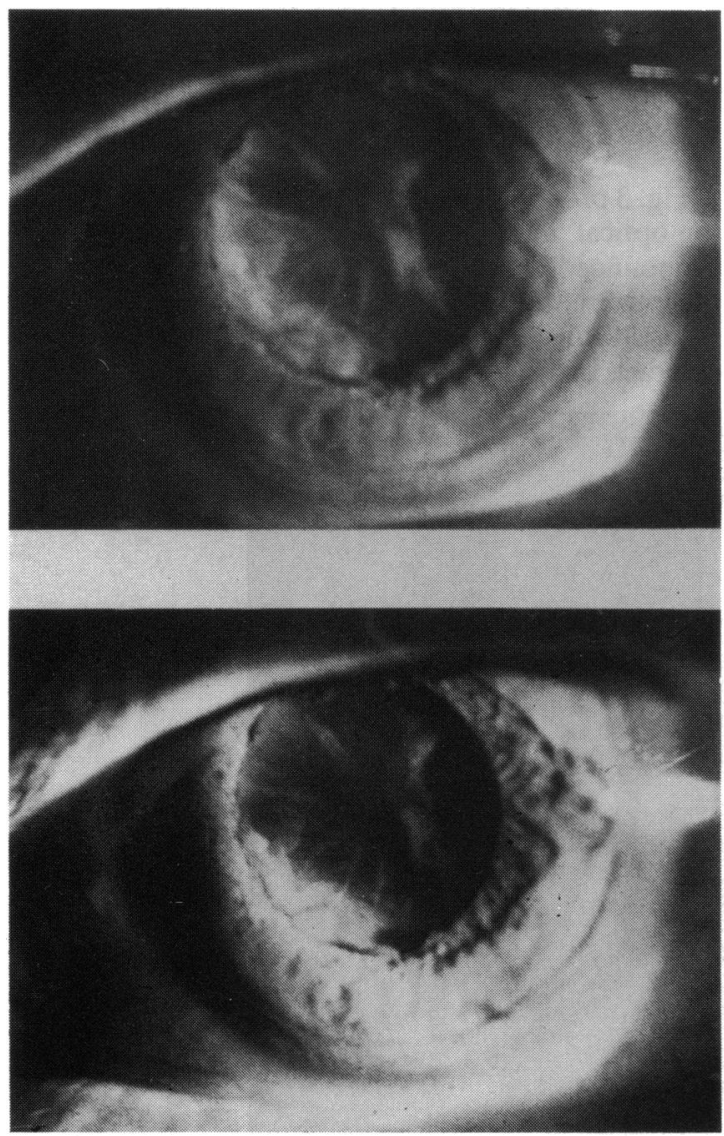

Fig. 8A tinuities: if it did, then the rotation of $Q$ should be without effect on the distribution of the light in the nucleus.

Fig. 5 shows a dense nuclear (brunescent) cataract. The two bottom pictures indicate the presence of clearly visible discontinuities, made manifest by the
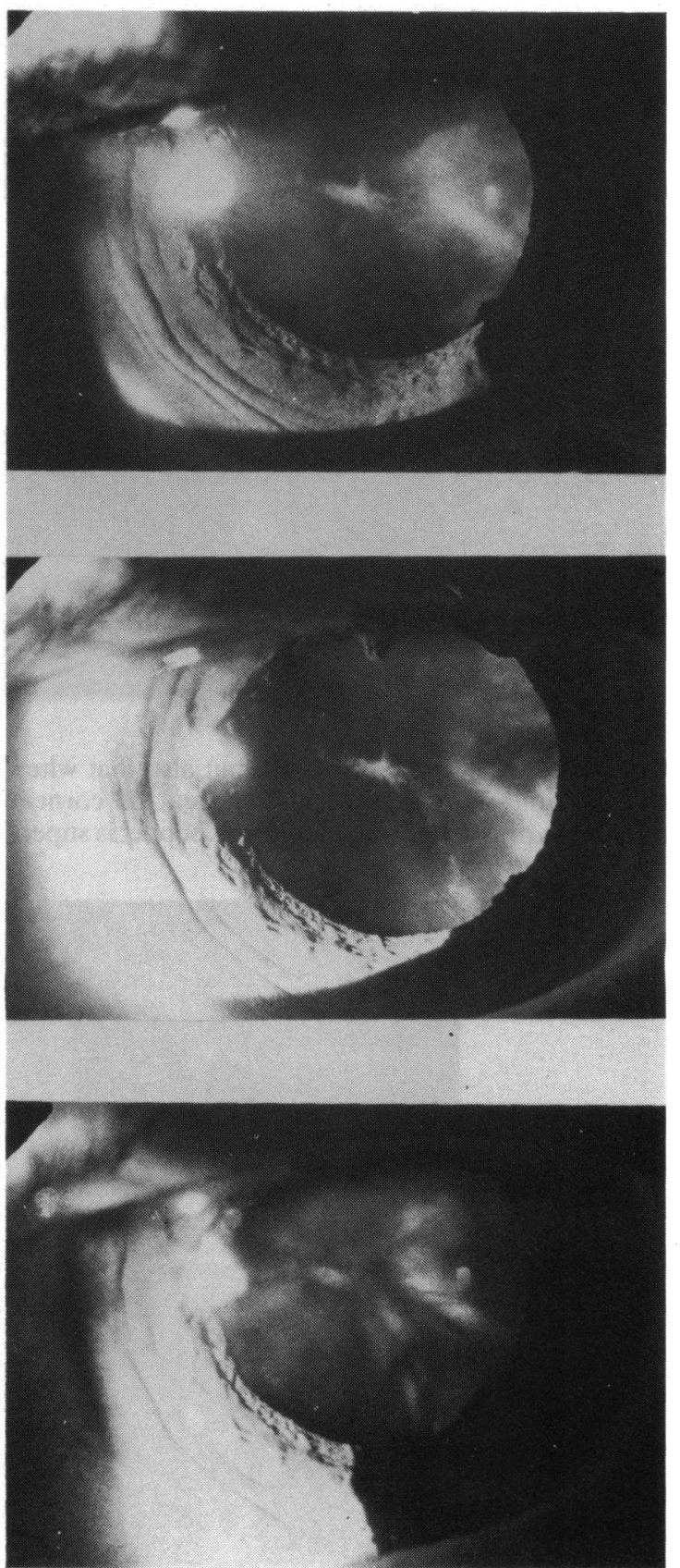

Fig. 8B 
appropriate angular position of the $\mathrm{Q}$ plate. Note their appearance in the side and frontal views respectively.

Fig. 6 highlights the increase in contrast obtainable in a case of generalised scatter and cuneiform cataract, which may be of value in monitoring progress in either direction.

Fig. 7 also illustrates the extent to which the presence of the 'Maltese cross' (arrows) may make measurement of discontinuties a realistic proposition.

Figs. 8A, B. These sequences refer to the left and right eyes of one patient. Fig. $8 \mathrm{~A}$ demonstrates the increase in contrast and, in addition to doing this, Fig. 8B shows that detail, including albumin deposits, can be revealed at different depths in the lens.

An example of a coronal cataract is shown in Fig. 9. Note how the reflexion from the posterior surface of the lens is virtually eliminated. Owing to the birefringence of the ocular media, the positions of $Q$ for minimising the intensities of the two principal reflexions are slightly dissimilar.

In Fig. 10 there are to be seen pepper-pot signs and

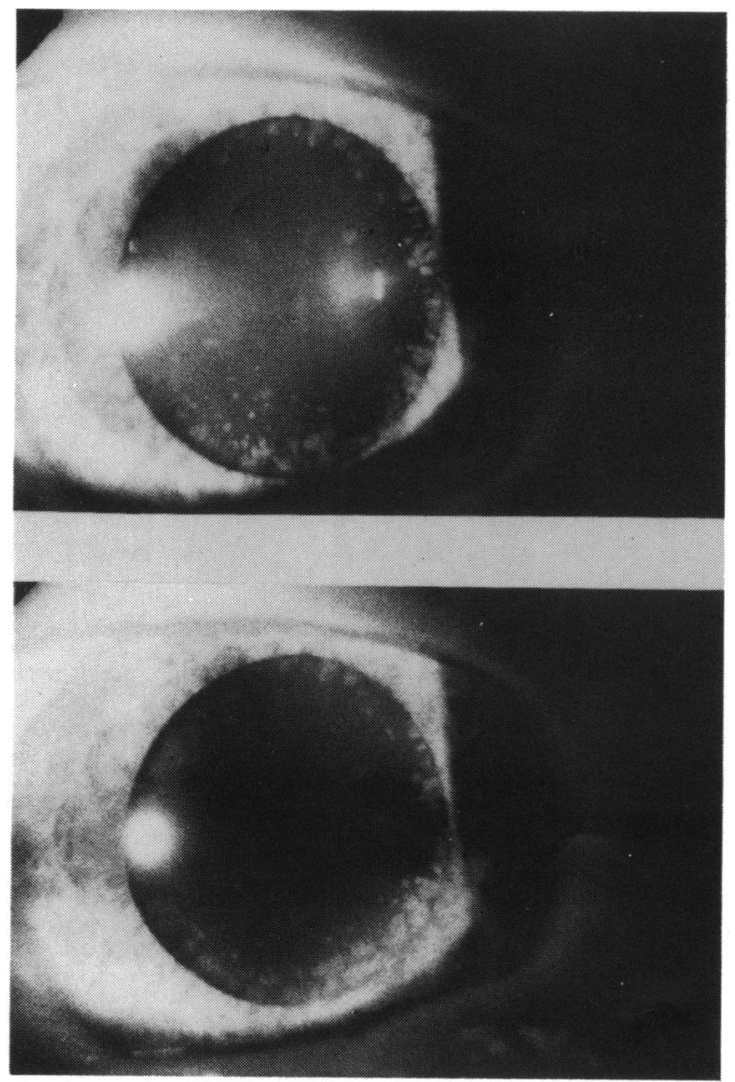

Fig. 9
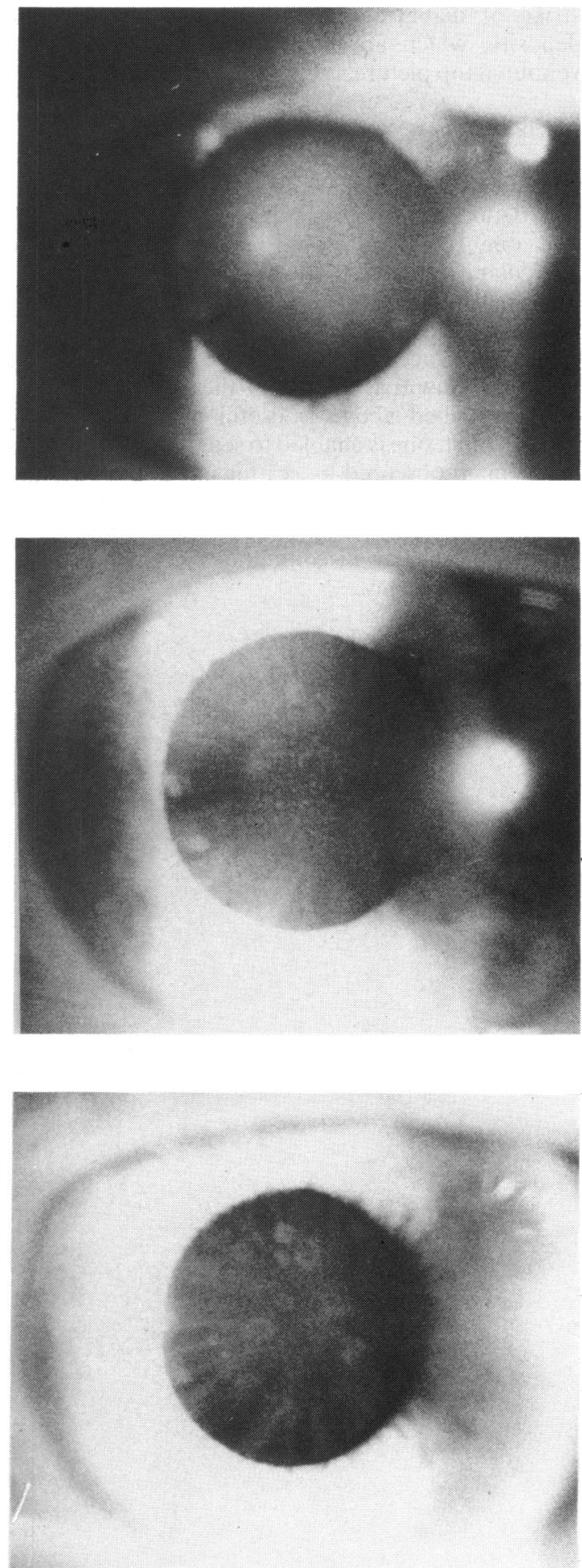

Fig. 10 
striae of diabetes (cf. Fisher ${ }^{8}$ ) and also calcium deposits, which are largely invisible in the conventional top picture.

\section{Discussion}

Several of the above illustrations support the suggestion deduced from observations on normal lenses ${ }^{4}$ that one has to distinguish apparent from real lenticular light scatter. The former can, but the latter cannot, be modified by the use of polarised light. The degree of modification is less marked in cases of nuclear cataract, but is rarely negligible. It would seem to follow that the use of the optical modifications described above is useful for the following reasons: first, one is enabled to see structural changes that remain obscured by scatter in normal viewing; secondly, such changes may facilitate a differential diagnosis; thirdly, the technique makes it possible to quantify lenticular discontinuities and to monitor their changes, if any.
I thank Professor R F Fisher for the loan of a Zeiss photo slit-lamp and helpful discussions, and Miss G M Villermet for technical assistance.

\section{References}

1 Zuckerman JL, Miller D, Dyes W, Keller M. Degradation of vision through a simulated cataract. Invest Ophthalmol Vis Sci 1973; 12: 213-24.

2 Wood RW. Physical Optics. New York: Macmillan, 1934.

3 Koeppe L. Das biophysikalisch-histologische Verhalten der lebenden Augengewebe unter normalen und pathologischen Bedingungen im polarisierten Lichte der Gullstrandschen Nernstspaltlampe. Graefes Arch Klin Exp Ophthalmol 1920; 102: 4-97.

4 Cope WT, Wolbarsht ML, Yamanashi BS. The corneal polarization cross. J Opt Soc Am 1978; 68: 1139-41.

5 Weale RA. Real light scatter in the human crystalline lens. Graefes Arch Klin Exp Ophthalmol in press.

6 Peli E. Circular polarizers enhance visibility of endothelium in the specular reflection biomicroscopy. Arch Ophthalmol 1985; 103: 670-2.

7 Velluz L, Legrand M, Grosjean M. Optical circular dichroism. London: Academic Press, 1965.

8 Fisher RF. The structure and function of basement membrane (lens capsule) in relation to diabetes and cataract. Trans Ophthalmol Soc UK 1985; 104: 755-9.

Accepted for publication 21 February 1986. 